\title{
ON THE GROWTH OF LINEAR RECURRENCES IN FUNCTION FIELDS CLEMENS FUCHS ${ }^{\otimes}$ and SEBASTIAN HEINTZE ${ }^{\circledR}$
}

(Received 21 July 2020; accepted 12 August 2020; first published online 9 November 2020)

\section{Abstract}

Let $\left(G_{n}\right)_{n=0}^{\infty}$ be a nondegenerate linear recurrence sequence whose power sum representation is given by $G_{n}=a_{1}(n) \alpha_{1}^{n}+\cdots+a_{t}(n) \alpha_{t}^{n}$. We prove a function field analogue of the well-known result in the number field case that, under some nonrestrictive conditions, $\left|G_{n}\right| \geq\left(\max _{j=1, \ldots, t}\left|\alpha_{j}\right|\right)^{n(1-\varepsilon)}$ for $n$ large enough.

2020 Mathematics subject classification: primary 11B37; secondary 11J87, 11R58.

Keywords and phrases: function fields, linear recurrences, $S$-units.

\section{Introduction}

Let $\left(G_{n}\right)_{n=0}^{\infty}$ be a nondegenerate linear recurrence sequence with power sum representation $G_{n}=a_{1}(n) \alpha_{1}^{n}+\cdots+a_{t}(n) \alpha_{t}^{n}$. This expression makes sense for a sequence $\left(G_{n}\right)_{n=0}^{\infty}$ taking values in any field $K$; the characteristic roots $\alpha_{i}$ as well as the coefficients of the polynomials $a_{i}$ then lie in a finite extension $L$ of $K$. In this paper $K$ is either a number field or a function field in one variable of characteristic zero. The nondegenerate condition means in the number field case that no ratio $\alpha_{i} / \alpha_{j}$ for $i \neq j$ is a root of unity and in the function field case that no ratio $\alpha_{i} / \alpha_{j}$ for $i \neq j$ is contained in the field of constants. In the number field case it is well known that, if $\max _{j=1, \ldots, t}\left|\alpha_{j}\right|>1$, then for any $\varepsilon>0$ the inequality

$$
\left|G_{n}\right| \geq\left(\max _{j=1, \ldots, t}\left|\alpha_{j}\right|\right)^{n(1-\varepsilon)}
$$

is satisfied for every sufficiently large $n$.

The purpose of this paper is to prove an analogous result in the case of a function field in one variable of characteristic zero. Thus we answer, in the setting we are

Supported by Austrian Science Fund (FWF): I4406.

(C) 2020 Australian Mathematical Publishing Association Inc. This is an Open Access article, distributed under the terms of the Creative Commons Attribution licence (http://creativecommons. org/licenses/by/4.0/), which permits unrestricted re-use, distribution, and reproduction in any medium, provided the original work is properly cited. 
working in, Open Question 3 in [11]; we are grateful to Shparlinski for bringing this paper to our attention. Firstly, we will prove a theorem which states an inequality for an arbitrary valuation in the splitting field $L$ of the characteristic polynomial belonging to the linear recurrence sequence. Secondly, we will derive a corollary for the special case of polynomial power sums. In this special case the inequality takes a form very similar to (1.1). At this point we make the following observation: Theorem 1 in [6] already implies that under some nondegeneracy conditions the degree of polynomials in a linear recurrence sequence with polynomial roots cannot be bounded and therefore must grow to infinity as $n$ does. But this theorem does not say how fast it must grow. In the present paper we will give a bound depending on $n$ for the minimal possible degree of $G_{n}$.

The number field case is often mentioned (see [3, 8] or more recently [1, 2]), but it is not that easy to access a proof of it (see [10] or the formulation in [9]). So we give a complete proof based on results of Evertse and Schmidt in the Appendix. By doing so we contribute to the goal that well-known facts should be fully accessible with proof following van der Poorten's wise statement in [9] that 'all too frequently, the well known is [often] not generally known, let alone known well'.

\section{Results and notations}

Throughout the paper we denote by $K$ a function field in one variable over $\mathbb{C}$. By $L$ we usually denote a finite algebraic extension of $K$. For the convenience of the reader we give a short summary of the notion of valuations that can also be found in [4]. For $c \in \mathbb{C}$ and $f(x) \in \mathbb{C}(x)$, where $\mathbb{C}(x)$ is the rational function field over $\mathbb{C}$, we denote by $v_{c}(f)$ the unique integer such that $f(x)=(x-c)^{v_{c}(f)} p(x) / q(x)$ with $p(x), q(x) \in \mathbb{C}[x]$ such that $p(c) q(c) \neq 0$. Further, we write $v_{\infty}(f)=\operatorname{deg} q-\operatorname{deg} p$ if $f(x)=p(x) / q(x)$. These functions $v: \mathbb{C}(x) \rightarrow \mathbb{Z}$ are up to equivalence all valuations in $\mathbb{C}(x)$. If $v_{c}(f)>0$, then $c$ is called a zero of $f$, and if $v_{c}(f)<0$, then $c$ is called a pole of $f$, where $c \in \mathbb{C} \cup\{\infty\}$. For a finite extension $K$ of $\mathbb{C}(x)$ each valuation in $\mathbb{C}(x)$ can be extended to no more than $[K: \mathbb{C}(x)]$ valuations in $K$. This again gives up to equivalence all valuations in $K$. Both in $\mathbb{C}(x)$ as well as in $K$ the sum formula

$$
\sum_{v} v(f)=0
$$

holds, where the sum is taken over all valuations in the relevant function field. Valuations have the properties $v(f g)=v(f)+v(g)$ and $v(f+g) \geq \min (v(f), v(g))$ for all $f, g \in K$. Each valuation in a function field corresponds to a place and vice versa. The places can be thought of as the equivalence classes of valuations. For more information about valuations and places we refer to [13].

For any power sum $G_{n}=a_{1}(n) \alpha_{1}^{n}+\cdots+a_{t}(n) \alpha_{t}^{n}$ with $a_{j}(n)=\sum_{k=0}^{m_{j}} a_{j k} n^{k}$ and any valuation $\mu$ (in a function field $L / K$ containing the $\alpha_{j}$ and the coefficients of the $a_{j}$ ) we 
have the trivial bound

$$
\begin{aligned}
\mu\left(G_{n}\right) & =\mu\left(a_{1}(n) \alpha_{1}^{n}+\cdots+a_{t}(n) \alpha_{t}^{n}\right) \geq \min _{j=1, \ldots, t} \mu\left(a_{j}(n) \alpha_{j}^{n}\right) \\
& \geq \min _{j=1, \ldots, t} \mu\left(a_{j}(n)\right)+\min _{j=1, \ldots, t} \mu\left(\alpha_{j}^{n}\right) \\
& \geq \min _{j=1, \ldots, t, t=0, \ldots, m_{j}} \mu\left(a_{j k} n^{k}\right)+n \cdot \min _{j=1, \ldots, t} \mu\left(\alpha_{j}\right) \\
& =\min _{\substack{j=1, \ldots, t \\
k=0, \ldots, m_{j}}} \mu\left(a_{j k}\right)+n \cdot \min _{j=1, \ldots, t} \mu\left(\alpha_{j}\right)=\widetilde{C}+n \cdot \min _{j=1, \ldots, t} \mu\left(\alpha_{j}\right) .
\end{aligned}
$$

Our main result is now the following theorem which gives a bound in the other direction.

THEOREM 2.1. Let $\left(G_{n}\right)_{n=0}^{\infty}$ be a nondegenerate linear recurrence sequence taking values in $K$ with power sum representation $G_{n}=a_{1}(n) \alpha_{1}^{n}+\cdots+a_{t}(n) \alpha_{t}^{n}$. Let $L=K\left(\alpha_{1}, \ldots, \alpha_{t}\right)$ be the splitting field of the characteristic polynomial of that sequence and let $\mu$ be a valuation on $L$. Then there is an effectively computable constant $C$, independent of $n$, such that, for every sufficiently large $n$, the inequality

$$
\mu\left(G_{n}\right) \leq C+n \cdot \min _{j=1, \ldots, t} \mu\left(\alpha_{j}\right)
$$

holds.

For the special case of a linear recurrence sequence of complex polynomials having complex polynomials as characteristic roots we get the following lower bound for the degree of the $n$th member of the sequence.

COROLLARY 2.2. Let $\left(G_{n}\right)_{n=0}^{\infty}$ be a nondegenerate linear recurrence sequence of polynomials in $\mathbb{C}[x]$ with power sum representation $G_{n}=a_{1}(n) \alpha_{1}^{n}+\cdots+a_{t}(n) \alpha_{t}^{n}$ such that $\alpha_{1}, \ldots, \alpha_{t} \in \mathbb{C}[x]$. Then there is an effectively computable constant $C$, independent of $n$, such that, for every sufficiently large $n$, the inequality

$$
\operatorname{deg} G_{n} \geq n \cdot \max _{j=1, \ldots, t} \operatorname{deg} \alpha_{j}-C
$$

holds.

In the case of a binary recurrence sequence of polynomials, that is, $t=2$ in Corollary 2.2, one can use Mason's function field $a b c$ theorem (see [7]) to show that the number of distinct zeros of $G_{n}$ must go to infinity as $n$ does. By considering this in slightly more detail, the number of distinct zeros of $G_{n}$ can be bounded above (trivially) and below (by means of the $a b c$ theorem) both by linear polynomials in $n$.

It would be interesting to prove a function field variant of Corollary 3.1 in [1]. However, because of Lemma 3.1, which is based on Dirichlet's classical approximation theorem, we are not (yet) able to prove such a statement.

In the proof given in the next section we will apply the following special case of Theorem 1 in [6]. 
LEMMA 2.3. Let $K$ be as above and $L$ be a finite extension of $K$ of genus $\mathfrak{g}$. Furthermore, let $\alpha_{1}, \ldots, \alpha_{d} \in L^{*}$ with $d \geq 2$ be such that $\alpha_{i} / \alpha_{j} \notin \mathbb{C}^{*}$ for each pair of subscripts $i, j$ with $1 \leq i<j \leq d$. Moreover, for every $i=1, \ldots, d$, let $\pi_{i 1}, \ldots, \pi_{i r_{i}} \in L$ be $r_{i}$ linearly independent elements over $\mathbb{C}$. Put

$$
q=\sum_{i=1}^{d} r_{i} .
$$

Then, for every $n \in \mathbb{N}$ such that

$$
\left\{\pi_{i l} \alpha_{i}^{n}: l=1, \ldots, r_{i}, i=1, \ldots, d\right\}
$$

is linearly dependent over $\mathbb{C}$, but no proper subset of this set is linearly dependent over $\mathbb{C}$, we have

$$
n \leq C=C\left(q, \mathfrak{g}, \pi_{i l}, \alpha_{i}: l=1, \ldots, r_{i}, i=1, \ldots, d\right) .
$$

The proof will also make use of height functions in function fields. Let us therefore define the height of an element $f \in L^{*}$ by

$$
\mathcal{H}(f):=-\sum_{v} \min (0, v(f))=\sum_{v} \max (0, v(f)),
$$

where the sum is taken over all valuations in the function field $L / \mathbb{C}$. For every $z \in$ $L \backslash \mathbb{C}$,

$$
\begin{aligned}
\mathcal{H}(z) & =\sum_{v} \max (0, v(z))=\sum_{P} \max \left(0, v_{P}(z)\right) \\
& =\operatorname{deg} \sum_{P} \max \left(0, v_{P}(z)\right) P=\operatorname{deg}(z)_{0}=[L: \mathbb{C}(z)]=\operatorname{deg}_{\mathbb{C}}(z),
\end{aligned}
$$

by Theorem I.4.11 in [13], where we have used the fact that all places have degree one since we are working over $\mathbb{C}$ (instead of the height, one can use $\operatorname{deg}_{\mathbb{C}}(z)=[L: \mathbb{C}(z)]$ as in [14]). Additionally, we define $\mathcal{H}(0)=\infty$. This height function satisfies some basic properties that are listed in the next lemma which is proven in [5].

LEMMA 2.4. Let $\mathcal{H}$ denote the height on $L / \mathbb{C}$ as above. Then, for $f, g \in L^{*}$ :

(a) $\mathcal{H}(f) \geq 0$ and $\mathcal{H}(f)=\mathcal{H}(1 / f)$;

(b) $\mathcal{H}(f)-\mathcal{H}(g) \leq \mathcal{H}(f+g) \leq \mathcal{H}(f)+\mathcal{H}(g)$;

(c) $\mathcal{H}(f)-\mathcal{H}(g) \leq \mathcal{H}(f g) \leq \mathcal{H}(f)+\mathcal{H}(g)$;

(d) $\mathcal{H}\left(f^{n}\right)=|n| \cdot \mathcal{H}(f)$;

(e) $\mathcal{H}(f)=0 \Longleftrightarrow f \in \mathbb{C}^{*}$;

(f) $\mathcal{H}(A(f))=\operatorname{deg} A \cdot \mathcal{H}(f)$ for any $A \in \mathbb{C}[T] \backslash\{0\}$.

We will also use the following function field analogue of the Schmidt subspace theorem.

Proposition 2.5 (Zannier [14]). Let $F / \mathbb{C}$ be a function field in one variable and of genus g. Let $\varphi_{1}, \ldots, \varphi_{n} \in F$ be linearly independent over $\mathbb{C}$ and let $r \in\{0,1, \ldots, n\}$. 
Let $S$ be a finite set of places of $F$ containing all the poles of $\varphi_{1}, \ldots, \varphi_{n}$ and all the zeros of $\varphi_{1}, \ldots, \varphi_{r}$. Put $\sigma=\sum_{i=1}^{n} \varphi_{i}$. Then

$$
\sum_{v \in S}\left(v(\sigma)-\min _{i=1, \ldots, n} v\left(\varphi_{i}\right)\right) \leq\left(\begin{array}{l}
n \\
2
\end{array}\right)(|S|+2 \mathfrak{g}-2)+\sum_{i=r+1}^{n} \mathcal{H}\left(\varphi_{i}\right) .
$$

\section{Proofs}

Proof of Theorem 2.1. Denote the coefficients of the polynomial $a_{j}(n) \in L[n]$ by $a_{j 0}, a_{j 1}, \ldots, a_{j m_{j}}$ where $m_{j}$ is the degree of $a_{j}(n)$. So

$$
a_{j}(n)=\sum_{k=0}^{m_{j}} a_{j k} n^{k}
$$

First assume that the recurrence sequence is of the shape $G_{n}=a_{1}(n) \alpha_{1}^{n}$. Using Lemma 2.4,

$$
\begin{aligned}
\mu\left(G_{n}\right)=\mu\left(a_{1}(n)\right)+n \mu\left(\alpha_{1}\right) & \leq \mathcal{H}\left(a_{1}(n)\right)+n \mu\left(\alpha_{1}\right) \\
& \leq \sum_{k=0}^{m_{1}} \mathcal{H}\left(a_{1 k} n^{k}\right)+n \mu\left(\alpha_{1}\right)=\sum_{k=0}^{m_{1}} \mathcal{H}\left(a_{1 k}\right)+n \mu\left(\alpha_{1}\right) .
\end{aligned}
$$

Thus from now on we can assume that $t \geq 2$. Let $\pi_{j 1}, \ldots, \pi_{j k_{j}}$ be a maximal $\mathbb{C}$-linear independent subset of $a_{j 0}, a_{j 1}, \ldots, a_{j m_{j}}$. Then we can write the sequence as

$$
G_{n}=\sum_{j=1}^{t}\left(\sum_{i=1}^{k_{j}} b_{j i}(n) \pi_{j i}\right) \alpha_{j}^{n}
$$

with polynomials $b_{j i}(n) \in \mathbb{C}[n]$. Since $a_{j}(n)$ is not the zero polynomial, there is for each $j$ at least one index $i$ such that $b_{j i}(n)$ is not the zero polynomial. Without loss of generality we can assume that no $b_{j i}(n)$ is the zero polynomial since otherwise we can throw out all zero polynomials and renumber the remaining terms. It does not matter whether all $\pi_{j i}$ occur in the sum or not. Moreover, we assume that $n$ is large enough such that $b_{j i}(n) \neq 0$ for all $j, i$.

Consider as a next step the set

$$
M:=\left\{\pi_{j i} \alpha_{j}^{n}: i=1, \ldots, k_{j}, j=1, \ldots, t\right\} .
$$

We intend to apply Lemma 2.3 . If $M$ is linearly dependent over $\mathbb{C}$, then we choose a minimal linearly dependent subset $\widetilde{M}$ of $M$, that is, a linearly dependent subset $\widetilde{M}$ with the property that no proper subset of $\widetilde{M}$ is linearly dependent. Let $\widetilde{G}_{n}$ be the linear recurrence sequence associated with this subset $\widetilde{M}$, that is,

$$
\widetilde{G_{n}}=\sum_{j=1}^{s}\left(\sum_{i=1}^{\widetilde{k_{j}}} b_{j i}(n) \pi_{j i}\right) \alpha_{j}^{n}
$$


for $s \leq t$ and after a suitable renumbering of the summands. Since $\pi_{j 1}, \ldots, \pi_{j k_{j}}$ are C-linearly independent we have $s \geq 2$. Applying Lemma 2.3 to

$$
\widetilde{M}:=\left\{\pi_{j i} \alpha_{j}^{n}: i=1, \ldots, \widetilde{k_{j}}, j=1, \ldots, s\right\}
$$

gives an upper bound for $n$. Thus for $n$ large enough this subset $\widetilde{M}$ of $M$ cannot be linearly dependent. Because of the fact that there are only finitely many subsets of $M$, for $n$ large enough the set $M$ must be linearly independent.

We assume from here on that $n$ is large enough such that $M$ is linearly independent. For each fixed $n$ we have $b_{j i}(n) \in \mathbb{C}^{*}$. Thus the set

$$
M^{\prime}:=\left\{b_{j i}(n) \pi_{j i} \alpha_{j}^{n}: i=1, \ldots, k_{j}, j=1, \ldots, t\right\} .
$$

is linearly independent over $\mathbb{C}$ and contains for each $j=1, \ldots, t$ at least one element. Let $S$ be a finite set of places of $L$ containing all zeros and poles of $\alpha_{j}$ for $j=1, \ldots, t$ and of the nonzero $a_{j i}$ for $j=1, \ldots, t$ and $i=1, \ldots, m_{j}$ as well as $\mu$ and the places lying over $\infty$. Now applying Proposition 2.5 yields

$$
\sum_{v \in S}\left(v\left(G_{n}\right)-\min _{\substack{j=1, \ldots, t \\
i=1, \ldots, k_{j}}} v\left(b_{j i}(n) \pi_{j i} \alpha_{j}^{n}\right)\right) \leq\left(\begin{array}{c}
\sum_{j=1}^{t} k_{j} \\
2
\end{array}\right)(|S|+2 \mathfrak{g}-2)=: C_{1}
$$

and, since each summand in the sum on the left-hand side is nonnegative,

$$
\mu\left(G_{n}\right)-\min _{\substack{j=1, \ldots, t \\ i=1, \ldots, k_{j}}} \mu\left(b_{j i}(n) \pi_{j i} \alpha_{j}^{n}\right) \leq C_{1} .
$$

Therefore for all $j_{0}=1, \ldots, t$ and $i_{0}=1, \ldots, k_{j_{0}}$,

$$
\begin{aligned}
\mu\left(G_{n}\right) & \leq C_{1}+\min _{\substack{j=1, \ldots, t \\
i=1, \ldots, k_{j}}} \mu\left(b_{j i}(n) \pi_{j i} \alpha_{j}^{n}\right) \\
& \leq C_{1}+\mu\left(b_{j_{0} i_{0}}(n) \pi_{j_{0} i_{0}} \alpha_{j_{0}}^{n}\right) \\
& =C_{1}+\mu\left(\pi_{j_{0} i_{0}}\right)+n \mu\left(\alpha_{j_{0}}\right) \\
& \leq C_{1}+\max _{\substack{j=1, \ldots, t \\
i=0, \ldots, m_{j}, a_{j i} \neq 0}} \mu\left(a_{j i}\right)+n \mu\left(\alpha_{j_{0}}\right) \\
& \leq C_{1}+\max _{j=1, \ldots, t} \mathcal{H}\left(a_{j i}\right)+n \mu\left(\alpha_{j_{0}}\right) \\
& =C_{2}+n \mu\left(\alpha_{j_{0}}\right) ., \ldots, m_{j}, a_{j i} \neq 0
\end{aligned}
$$

Since this holds for all $j_{0}=1, \ldots, t$,

$$
\mu\left(G_{n}\right) \leq C_{2}+n \cdot \min _{j=1, \ldots, t} \mu\left(\alpha_{j}\right) .
$$

Proof of Corollary 2.2. We can apply Theorem 2.1 with $L=K=\mathbb{C}(x)$ and $\mu=v_{\infty}$. This yields

$$
-\operatorname{deg} G_{n}=v_{\infty}\left(G_{n}\right) \leq C+n \cdot \min _{j=1, \ldots, t} v_{\infty}\left(\alpha_{j}\right)=C-n \cdot \max _{j=1, \ldots, t} \operatorname{deg} \alpha_{j}
$$

which immediately implies the inequality in question. 


\section{Appendix A. The number field case}

In this appendix we will give a proof of the following theorem.

THEOREM A.1. Let $\left(G_{n}\right)_{n=0}^{\infty}$ be a nondegenerate linear recurrence sequence taking values in a number field $K$ and let $G_{n}=a_{1}(n) \alpha_{1}^{n}+\cdots+a_{t}(n) \alpha_{t}^{n}$ with algebraic integers $\alpha_{1}, \ldots, \alpha_{t}$ be its power sum representation satisfying $\max _{j=1, \ldots, t}\left|\alpha_{j}\right|>1$. Denote by $|\cdot|$ the usual absolute value on $\mathbb{C}$. Then, for any $\varepsilon>0$, the inequality

$$
\left|G_{n}\right| \geq\left(\max _{j=1, \ldots, t}\left|\alpha_{j}\right|\right)^{n(1-\varepsilon)}
$$

is satisfied for every sufficiently large $n$.

Note that this result is not effective in the sense that we do not give a bound $n_{0}$ such that the inequality is satisfied for all $n$ greater than $n_{0}$. If we were to look more precisely at the limitations placed on $n$ in the proof given below, it would be possible to give an (admittedly) rather complicated upper bound on the number of exceptions. This bound would have the following form: if $n / \log n>B_{1}$, then there are at most $B_{2}$ values of $n$ for which the inequality is not valid. Since the explicit constants are not so enlightening we will not calculate them in detail.

From here on $K$ will denote a number field. In the proof we will need three auxiliary results which are listed below. The first one is a result of Schmidt.

LeMmA A.2 (Schmidt [12]). Suppose that $\left(G_{n}\right)_{n \in \mathbb{Z}}$ is a nondegenerate linear recurrence sequence of complex numbers, whose characteristic polynomial has $k$ distinct roots of multiplicity at most $a$. Then the number of solutions $n \in \mathbb{Z}$ of the equation

$$
G_{n}=0
$$

can be bounded above by

$$
c(k, a)=e^{\left(7 k^{a}\right)^{8 k^{a}}} .
$$

The second is a result of Evertse. We use the notation

$$
\|\mathbf{x}\|=\max _{\substack{k=0, \ldots, t \\ i=1, \ldots, D}}\left|\sigma_{i}\left(x_{k}\right)\right|
$$

with $\left\{\sigma_{1}, \ldots, \sigma_{D}\right\}$ the set of all embeddings of $K$ in $\mathbb{C}$ and $\mathbf{x}=\left(x_{0}, x_{1}, \ldots, x_{t}\right)$. Moreover, we denote by $O_{K}$ the ring of integers in $K$.

LEMMA A.3 (Evertse [3]). Let $t$ be a nonnegative integer and $S$ a finite set of places in $K$, containing all infinite places. Then for every $\varepsilon>0$ a constant $C$ exists, depending only on $\varepsilon, S, K, t$ such that for each nonempty subset $T$ of $S$ and every vector $\mathbf{x}=\left(x_{0}, x_{1}, \ldots, x_{t}\right) \in O_{K}^{t+1}$ with

$$
x_{i_{0}}+x_{i_{1}}+\cdots+x_{i_{s}} \neq 0
$$


for each nonempty subset $\left\{i_{0}, i_{1}, \ldots, i_{s}\right\}$ of $\{0,1, \ldots, t\}$,

$$
\left(\prod_{k=0}^{t} \prod_{v \in S}\left\|x_{k}\right\|_{v}\right) \prod_{v \in T}\left\|x_{0}+x_{1}+\cdots+x_{t}\right\|_{v} \geq C\left(\prod_{v \in T} \max _{k=0, \ldots, t}\left\|x_{k}\right\|_{v}\right)\|\mathbf{x}\|^{-\varepsilon} .
$$

Furthermore, we will need the following lemma which also can be found in [3].

Lemma A.4. Suppose $K$ is a number field of degree $D$, let $f(X) \in K[X]$ be a polynomial of degree $m$ and $T$ a nonempty set of primes on $K$. Then there exists $a$ positive constant $c$, depending only on $K, f$ such that for all $r \in \mathbb{Z}$ with $r \neq 0$ and $f(r) \neq 0$,

$$
\begin{aligned}
c^{-1}|r|^{-D m} \leq\left(\prod_{v} \max \left(1,\|f(r)\|_{v}\right)\right)^{-1} & \leq \prod_{v \in T}\|f(r)\|_{v} \\
& \leq \prod_{v} \max \left(1,\|f(r)\|_{v}\right) \leq c|r|^{D m} .
\end{aligned}
$$

Proof OF TheOREM A.1. Since the characteristic roots $\alpha_{j}$ of $G_{n}$ are algebraic integers we can find a nonzero integer $z$ such that $z a_{j}(n) \alpha_{j}^{n}$ are algebraic integers for all $j=$ $1, \ldots, t$ and all $n \in \mathbb{N}$. Set $L=K\left(\alpha_{1}, \ldots, \alpha_{t}\right)$, the splitting field of the characteristic polynomial of the sequence $G_{n}$. Choose $S$ as a finite set of places in $L$ containing all infinite places as well as all places such that $\alpha_{1}, \ldots, \alpha_{t}$ are $S$-units. Let $\mu$ be such that $\|\cdot\|_{\mu}=|\cdot|$ is the usual absolute value on $\mathbb{C}$. In particular, $\mu \in S$. Further, define $T=\{\mu\}$.

As $G_{n}$ is nondegenerate, the sequence $\widetilde{G_{n}}=z G_{n}$ is also nondegenerate. Therefore by Lemma A.2, for $n$ large enough,

$$
z a_{j_{1}}(n) \alpha_{j_{1}}^{n}+\cdots+z a_{j_{s}}(n) \alpha_{j_{s}}^{n} \neq 0
$$

for each non-empty subset $\left\{j_{1}, \ldots, j_{s}\right\}$ of $\{1, \ldots, t\}$. Thus we can apply Lemma A.3 and get

$$
\left(\prod_{j=1}^{t} \prod_{v \in S}\left\|z a_{j}(n) \alpha_{j}^{n}\right\|_{\nu}\right)\left|z G_{n}\right| \geq C \max _{j=1, \ldots, t}\left|z a_{j}(n) \alpha_{j}^{n}\right|\|z \mathbf{x}\|^{-\varepsilon}
$$

for $\mathbf{x}=\left(a_{1}(n) \alpha_{1}^{n}, \ldots, a_{t}(n) \alpha_{t}^{n}\right)$. Without loss of generality, we can assume that $\left|\alpha_{1}\right|=$ $\max _{j=1, \ldots, t}\left|\alpha_{j}\right|$. Since $z$ is a fixed integer and the $\alpha_{j}$ are $S$-units, we can rewrite this as

$$
\begin{aligned}
\left(\prod_{j=1}^{t} \prod_{v \in S}\left\|a_{j}(n)\right\|_{\nu}\right)\left|G_{n}\right| & \geq C_{1} \max _{j=1, \ldots, t}\left|a_{j}(n) \alpha_{j}^{n}\right|\|\mathbf{x}\|^{-\varepsilon} \\
& \geq C_{1}\left|a_{1}(n) \alpha_{1}^{n}\right|\|\mathbf{x}\|^{-\varepsilon}=C_{1}\left|a_{1}(n)\right|\left|\alpha_{1}\right|^{n}\|\mathbf{x}\|^{-\varepsilon} .
\end{aligned}
$$

In preparation for the next step, note that there exists a positive constant $A$ such that

$$
\max _{\substack{j=1, \ldots, t \\ i=1, \ldots, D}}\left|\sigma_{i}\left(\alpha_{j}\right)\right| \leq A \cdot\left|\alpha_{1}\right| \text {. }
$$


We decompose $\varepsilon=\gamma \cdot \delta$ with small $\delta$ and $A^{\gamma} \leq\left|\alpha_{1}\right|$. This gives the estimates

$$
\begin{aligned}
\|\mathbf{x}\| & =\max _{\substack{j=1, \ldots, t \\
i=1, \ldots, D}}\left|\sigma_{i}\left(a_{j}(n) \alpha_{j}^{n}\right)\right|=\max _{\substack{j=1, \ldots, t \\
i=1, \ldots, D}}\left|\sigma_{i}\left(a_{j}(n)\right) \sigma_{i}\left(\alpha_{j}\right)^{n}\right| \\
& \leq \max _{\substack{j=1, \ldots, t \\
i=1, \ldots, D}}\left|\sigma_{i}\left(a_{j}(n)\right)\right| \cdot \max _{\substack{j=1, \ldots, t \\
i=1, \ldots, D}}\left|\sigma_{i}\left(\alpha_{j}\right)\right|^{n} \\
& \leq C_{2} n^{m} \cdot \max _{\substack{j=1, \ldots, t \\
i=1, \ldots, D}}\left|\sigma_{i}\left(\alpha_{j}\right)\right|^{n} \leq C_{2} n^{m} A^{n}\left|\alpha_{1}\right|^{n},
\end{aligned}
$$

with $m=\max _{j=1, \ldots, t} \operatorname{deg} a_{j}$, and

$$
\|\mathbf{x}\|^{\varepsilon} \leq C_{3} n^{m \varepsilon} A^{\gamma n \delta}\left|\alpha_{1}\right|^{n \varepsilon} \leq C_{3} n^{m \varepsilon}\left|\alpha_{1}\right|^{n(\varepsilon+\delta)} .
$$

Now we insert this into inequality (A.1), giving

$$
\left(\prod_{j=1}^{t} \prod_{v \in S}\left\|a_{j}(n)\right\|_{v}\right)\left|G_{n}\right| \geq C_{4}\left|a_{1}(n)\right|\left|\alpha_{1}\right|^{n} n^{-m \varepsilon}\left|\alpha_{1}\right|^{-n(\varepsilon+\delta)} \geq C_{5} n^{-m \varepsilon}\left|\alpha_{1}\right|^{n(1-\varepsilon-\delta)} .
$$

Applying Lemma A.4 to the product in the brackets on the left hand side gives the bound

$$
\prod_{j=1}^{t} \prod_{v \in S}\left\|a_{j}(n)\right\|_{v} \leq \prod_{j=1}^{t} C_{6}^{(j)} n^{D m} \leq C_{7} n^{t D m} .
$$

Altogether, for $n$ large enough,

$$
\left|G_{n}\right| \geq C_{8} n^{-t D m-m \varepsilon}\left|\alpha_{1}\right|^{n(1-\varepsilon-\delta)} .
$$

Hence, recalling that $\left|\alpha_{1}\right|=\max _{j=1, \ldots, t}\left|\alpha_{j}\right|$, for $n$ large enough,

$$
\left|G_{n}\right| \geq\left(\max _{j=1, \ldots, t}\left|\alpha_{j}\right|\right)^{n(1-\widetilde{\varepsilon})} .
$$

This proves the theorem.

\section{References}

[1] S. Akiyama, J.-H. Evertse and A. Pethő, 'On nearly linear recurrence sequences' (English summary), in: Number Theory - Diophantine Problems, Uniform Distribution and Applications (Springer, Cham, 2017), 1-24.

[2] Y. Bugeaud and H. Kaneko, 'On perfect powers in linear recurrence sequences of integers', Kyushu J. Math. 73 (2019), 221-227.

[3] J.-H. Evertse, 'On sums of S-units and linear recurrences', Comp. Math. 53(2) (1984), 225-244.

[4] C. Fuchs and S. Heintze, 'Perfect powers in polynomial power sums', Preprint, 2019, arXiv: 1912.10033.

[5] C. Fuchs, C. Karolus and D. Kreso, 'Decomposable polynomials in second order linear recurrence sequences', Manuscripta Math. 159(3) (2019), 321-346.

[6] C. Fuchs and A. Pethő, 'Effective bounds for the zeros of linear recurrences in function fields', $J$. Théor. Nombres Bordeaux 17 (2005), 749-766.

[7] R. C. Mason, Diophantine Equations over Function Fields, London Mathematical Society Lecture Notes, 96 (Cambridge University Press, Cambridge, 1984). 
[8] A. J. van der Poorten, 'Some problems of recurrent interest', Topics in Classical Number Theory, Vol. I, II (Budapest, 1981), Colloquia Mathematica Societatis Janos Bolyai, 34 (North-Holland, Amsterdam, 1984), 1265-1294.

[9] A. J. van der Poorten, 'Some facts that should be better known, especially about rational functions', Number Theory and Applications (Kluwer Academic, Dordrecht, 1989), 497-528.

[10] A. J. van der Poorten and H. P. Schlickewei, 'The growth conditions for recurrence sequences', Macquarie Mathematical Reports 82-0041 (1982) (Macquarie University, Sydney, 2019).

[11] A. J. van der Poorten and I. Shparlinski, 'On sequences of polynomials defined by certain recurrence relations', Acta Sci. Math. (Szeged) 61 (1995), 77-103.

[12] W. M. Schmidt, 'The zero multiplicity of linear recurrence sequences', Acta Math. 182 (1999), 243-282.

[13] H. Stichtenoth, Algebraic Function Fields and Codes, Universitext (Springer, Berlin, 1993).

[14] U. Zannier, 'On composite lacunary polynomials and the proof of a conjecture of Schinzel', Invent. Math. 174(1) (2008), 127-138.

CLEMENS FUCHS, Department of Mathematics,

University of Salzburg, Hellbrunnerstr. 34, A-5020 Salzburg, Austria e-mail: clemens.fuchs@sbg.ac.at

SEBASTIAN HEINTZE, Department of Mathematics, University of Salzburg, Hellbrunnerstr. 34, A-5020 Salzburg, Austria e-mail: sebastian.heintze@sbg.ac.at 